\title{
The effect of bilateral subthalamic nucleus deep brain stimulation (STN-DBS) on the acoustic and prosodic features in patients with Parkinson's disease: A study protocol for the first trial on Iranian patients
}

\author{
Fatemeh Majdinasab ${ }^{1}$, Ahmadreza Khatoonabadi ${ }^{1}$, Seyyedeh Maryam Khoddami ${ }^{*}{ }^{*}$, Amirhassan Habibi $^{2}$
}

Received: 16 Mar 2017

Published: 22 Dec 2017

\begin{abstract}
Background: The effect of subthalamic nucleus deep brain stimulation (STN-DBS) on the voice features in Parkinson's disease (PD) is controversial. No study has evaluated the voice features of PD underwent STN-DBS by the acoustic, perceptual, and patientbased assessments comprehensively. Furthermore, there is no study to investigate prosodic features before and after DBS in PD. The current study aimed to assess the efficacy of STN-DBS on the voice and prosodic features by acoustic, perceptual and self-reported evaluations in PD.

Methods and Analysis: An experimental prospective cohort pretest-posttest group has been designed to survey patients with PD candidates for STN-DBS surgery. All participants will be evaluated by a speech and language pathologist before and after surgery in four different conditions as follow: pre-surgery: Medication On/ Medication Off; post-surgery: Stimulation On/ Stimulation Off. To compare pre-surgery and post-surgery conditions paired- samples T Test or Wilcoxon signed-rank test will be used. A $95 \%$ confidence interval ( $p$ value of $<0.05$ ) will be considered to be statistically significant. This study protocol is approved by the Ethical Committee of Teheran University of Medical Sciences, and the results of the study will be published in a peer-reviewed journal and presented at national congresses.
\end{abstract}

Keywords: Parkinson's disease, Sub thalamic nucleus deep brain stimulation (STN-DBS), Acoustic, Perceptual, Voice, Prosody

Copyright $₫$ Iran University of Medical Sciences

Cite this article as: Majdinasab F, Khatoonabadi A, Khoddami SM, Habibi A. The effect of bilateral subthalamic nucleus deep brain stimulation (STN-DBS) on the accoustic and prosodic features in patients with Parkinson's disease: A study protocol for the first trial on Iranian patients. Med $J$ Islam Repub Iran. 2017 (22 Dec);31:118. https://doi.org/10.14196/mjiri.31.118

\section{Introduction}

Parkinson's disease (PD) is a hypokinetic movement disorder resulted from progressive loss of dopaminergic neurons in the brain stem. The patients with PD suffer from major motor symptoms such as rigidity, bradykinesia, resting tremor, postural instabilities as well as many speech disturbances called hypokinetic dysarthria $(1,2)$.

There are different treatment approaches including medical, behavioral, and surgical suggested for PD. Levodopa therapy is the oldest and most effective medical approach

Corresponding author: Dr Seyyedeh Maryam Khoddami, khoddami@tums.ac.ir

1. Department of Speech Therapy, School of Rehabilitation, Teheran University of Medical Sciences, Tehran, Iran.

2. Department of Neurology, Iran University of Medical Sciences, Tehran, Iran routinely used for all patients at any stage of the disease (3). In the recent decade, deep brain stimulation of the sub thalamic nucleus (STN-DBS) is accepted as an effective surgical treatment in carefully selected people with advanced $\mathrm{PD}(4,5)$. The STN-DBS reduces drug-induced dyskinesia and improves motor disabilities by inhibition of sub thalamic nucleus (STN) activities (6). Although motor symptoms are the main targets of DBS, non-motor symptoms (NMSs) such as speech characteristics have

$\uparrow$ What is "already known" in this topic:

Bilateral deep brain stimulation of the sub thalamic nucleus (STN-DBS) improves motor disabilities in patients suffering from PD but its effect on the voice and prosody is controversial. There is no evidence about effectiveness of STN-DBS on the speech parameters in Iranian patients undergone DBS surgery.

$\rightarrow$ What this article adds:

This study will evaluate the efficacy of bilateral STN-DBS on the voice features of PD by multidimensional assessments and will investigate both form and function of prosody simultaneously. Furthermore, the effect of STN-DBS approach will assess in Iranian PD patients for the first time. 
more significant impact on the quality of life of patients with PD rather than motor symptoms $(7,8)$. Some NMSs ameliorate after STN- DBS, but some of them remain unchanged or even worsen (9). Therefore, clinicians should consider NMSs following STN-DBS to ensure optimal outcomes.

Nearly $90 \%$ of PD patients experience hypokinetic dysarthria in the course of the PD worsened by progression of disease (10). Dysarthria resulted from abnormalities in all speech subsystems including respiration, phonation, articulation, resonation, and prosody $(2,11)$. Super subtlety of vocal folds movements makes them more vulnerable than other parts of the body; thus, laryngeal muscles show earlier and more alternations due to PD (12).

As a result, voice features such as pitch, loudness, and quality are affected more and sooner than other speech subsystems $(13,14)$. Prosody is the second important speech subsystem that that has close relations with the voice features (15). Although, prosody is defined as "linguistic use of verbal aspect of speech without consideration of segmental aspects", it plays some pragmatic, emotional, psychometric, and facilitator roles in communication (16). The prosody is affected in more than half speech deviations in PD. Therefore, prosodic disorders such as mono loudness, mono pitch, and reduced stress, short rushes of speech, inappropriate silences, and variable rate are usually documented in this condition (17). In fact, evaluation of voice and prosody is not only important for the diagnosis and prognosis of PD, but also it helps clinicians to identify efficacy of the treatment approaches such as STN-DBS.

The effect of STN-DBS on the voice features of PD is controversial $(3,18-22)$. There are some reasons of challenging results. Some of them may derive from insufficient outcome measures related to voice features (23-25). Another main source of challenge is likely inappropriate tools to evaluate speech characteristics (26). Moreover, there is no study to survive all acoustic, perceptual, and patient based dimensions of voice in PD after STN-DBS. During the last decade, some studies investigated the prosody after STN-DBS, but they just focused on the "prosody recognition" and "emotional prosody" $(27,28)$. All mentioned studies have evaluated "form features" of prosody while more functional approaches emphasized on the communication efficiency of prosody are preferred (29).

Despite of passing one decade from a first STN-DBS in Iran and high prevalence of handicap resulted from voice disabilities of Iranian patients with PD (30), there is no evidence about the effect of STN-DBS on the voice, prosody and voice related quality of life in Iranian persons suffering from PD. The present study is going to investigate the effect of bilateral STN-DBS on the voice and prosodic characteristics of Iranian patients with PD. Furthermore, because of levodopa therapy is done for all patients in all conditions (before and after DBS), probably effect of medication will be evaluated beside STN-DBS. We hypothesized that: 1) STN-DBS will impact on the acoustic features of voice, perceptual characteristics of overall severity of dysphonia, and expression of prosody;
2) Levodopa therapy will effect on the voice and prosodic features; 3) Voice related quality of life will be changed after stimulation.

\section{Methods}

\section{Study design and Setting}

This study will be experimental, prospective cohort pretest- posttest group design, which will be performed at the movement disorders clinic of Rasoul-E-Akram Hospital, Iran University of Medical Sciences, Tehran, Iran.

\section{Ethical Approval}

This study has been approved by the Institutional Review Board, School of Rehabilitation, and the Ethics Committee of Tehran University of Medical Sciences (IR.TUMS.VCR.REC.1395.375). All participants will sign the written consent form before initiation of sampling.

\section{Participants}

Patients will be selected from the movement disorders clinic of Rasoul-E-Akram Hospital of IUMS by convenience sampling. All attendants who are suffering from advanced PD and candidate for STN-DBS surgery will be referred by a movement disorder neurologist of surgery team to a Speech and Language Pathologist (SLP) for evaluating inclusion and exclusion criteria. Demographic characteristics, medical history, and patient's lifestyle will be obtained by a questionnaire during direct interview.

\section{Inclusion and Exclusion Criteria}

The inclusion criteria consist of: 1) a diagnosis of idiopathic PD based on the UK Parkinson's disease Society Brain Bank's clinical diagnostic criteria (31); 2) absence of other neurological diseases; 3 ) absence of cognitive or untreated psychiatric disorders according to the Montreal Cognitive Assessment (MoCA) and psychiatric assessment (32); 4) ability to write and read; 5) Farsi as the native language; and 6) no structural abnormalities in the vocal tract. The Patients will be excluded if they had a history of voice therapy (during a recent year), cancer, chemotherapy, and radiotherapy. Furthermore, the participants who get laryngitis and infection of upper respiratory system during the study will be excluded.

\section{Adverse Effects}

It should be noted that our voice and prosodic assessments are not invasive so, no side effects will be anticipated. However, there may be some side effects such as infection, and intra cerebral hemorrhage $(\mathrm{ICH})$ due to the invasive nature of DBS. These symptoms are diagnosed by a movement disorder neurologist according to the clinical and para-clinical evaluations. Data gathering will be postponed until the neurologist confirms all adverse effects have been removed.

\section{Outcome Measures}

Acoustic voice measurement: The acoustic outcome measures will be the fundamental frequency (F0), standard deviation of F0, jitter, shimmer, harmonic to noise 
ratio (HNR) which will be analyzed by Praat software (version 6.0.05) during vowel prolongations, reading, and spontaneous speech.

Auditory-perceptual voice assessment: The overall severity of dysphonia will be only voice perceptual outcome which will be evaluated by the Persian version of the Consensus Auditory Perceptual Evaluation of Voice (CAPE-V) named the ATSHA (33). This perceptual voice feature will be assessed during vowel prolongations, reading, and spontaneous speech. The CAPE-V is one of the most frequent auditory perceptual voice assessment tool which has high validity between other perceptual assessments (34).

Expressive prosodic assessment: The outcome measures of expressive prosody will be emotional prosody (affect), chunking, focus, turn-end, short item imitation, and long item imitation which will be evaluated by the computerized Persian version of the Profiling Elements of Prosody in Speech-Communication (PPEPS-C). The PPEPS-C is based on the psycholinguistic model and evaluates both "form and function" of prosody during several speech tasks (35).

Voice related quality of life assessment: The outcome measure of voice related quality of life will be total score of voice handicap which will be extracted through the Persian version of Voice Handicap Index (VHI) questionnaire (36). The VHI is the most widely used selfassessment tool which includes 30 questions and quantifies handicap due to voice disorders by the patient (37).

\section{Procedure}

All included patients with PD will be evaluated based on the outcome measures under two different conditions of medication on (Med. On) and medication off (Med. Off) two weeks before surgery. The "Med. On" condition is defined as clinical improvement reported by patients and movement disorder neurologist due to effective dose of levodopa. According to the literature, the "Med. Off" condition is defined as returned symptoms of PD resulted from 10-12 hours withholding medication (20). There will be a week interval between two assessment conditions of Med. On and Med. Off before the surgery.

The surgical procedure and contact localization will be performed as followed. At the first for anatomical targeting, the Leksell G stereotactic frame (Elekta Instruments AB, Stockholm, Sweden) with an attached magnetic resonance imaging (MRI) compatible localizer will be placed on the head of patients before MRI. The STN target will be visualized on MRI and will be directly targeted using Stereonauta software (Estudios e Investigaciones Neurologicas, S.L., Madrid, Spain). Moreover, atlas-based targeting and manual calculation will be used to define anatomical target (38).

Secondly, a quadripolar DBS electrode (model-3389, Medtronic Inc., Minneapolis, USA) will be implanted in the left and right sides of targets respectively. Clinical effects of stimulation will be monitored with wrist rigidity, hand tremor, and bradykinesia. The day after leads implementation, the neuro-stimulator (model Kinetra, Medtronic Inc., Minneapolis, USA) will be placed in the left infraclavicular subcutaneously. Finally, a postoperative brain CT scan will be done to rule out possible complications especially intra cerebral hemorrhage (ICH) in patients undergone STN-DBS(6).

At least one month after surgery when stimulation and motor characteristics stabilized according to the patients and movement disorder neurologist reports, the outcome measures will be evaluated under two different conditions of stimulation off (Stim. Off) and stimulation on (Stim. On). Both evaluations after STN-DBS will be done under Med. On condition.

The patients will be evaluated in a quiet room (noise level below 35dB) (39). A head-worn AKG microphone (model C250L) attached to a MSI- CR420 laptop will be used to record vowels and speech tasks for the acoustic and perceptual voice assessments. Mouth-to microphone distance was approximately $5 \mathrm{~cm}$ (40). Furthermore, the expressive prosody will be evaluated using the computerized version of the PPEPS-C. However, the patients will be asked to complete the VHI questionnaire only under Med. On condition before the surgery and Stim. On condition after the surgery. It should be noted that all measurements will be performed by a trained SLP in hypokinetic dysarthria between 9:00 and 12:00 A.M.

\section{Sample size calculation}

Due to no related study for estimating the effect size, a pilot study will be done to determine the sample size. To estimate sample size, the study will be set at $\alpha=0.05$ and a power of $80 \%$.

\section{Data Analysis}

Statistical analysis will be performed using SPSS 20.0. Descriptive statistics will be calculated for the clinical assessments before and after surgery. Before analytic statistics, Kolmogorov-Smirnov test will be used to determine data normality. To measure the effect of medication before the surgery, we will use paired- samples T Test or Wilcoxon signed-rank test to compare the outcome measures between Med. On and Med. Off conditions. After the surgery, the outcome measures will be compared between Med. On- Stim. On and Med. On- Stim. Off conditions by the similar tests to measure the effect of STNDBS. Furthermore, paired- samples T Test or Wilcoxon signed-rank test will be used to compare pre-surgery and post-surgery conditions. A 95\% confidence interval ( $p$ value of $<0.05$ ) will be considered to be statistically significant.

\section{Results}

Characteristics of the participants will be showed in a table like Table 1. Descriptive-analytic statistics associated with the acoustic and perceptual features of voice before and after surgery will be illustrated in a table like Table 2. Moreover, descriptive and analytic data of VHI will be showed and discussed in the manuscript. Descriptive-analytic statistics associated with the outcome measures of expressive prosody before and after STNDBS will be showed in a table similar to Table 3 . 


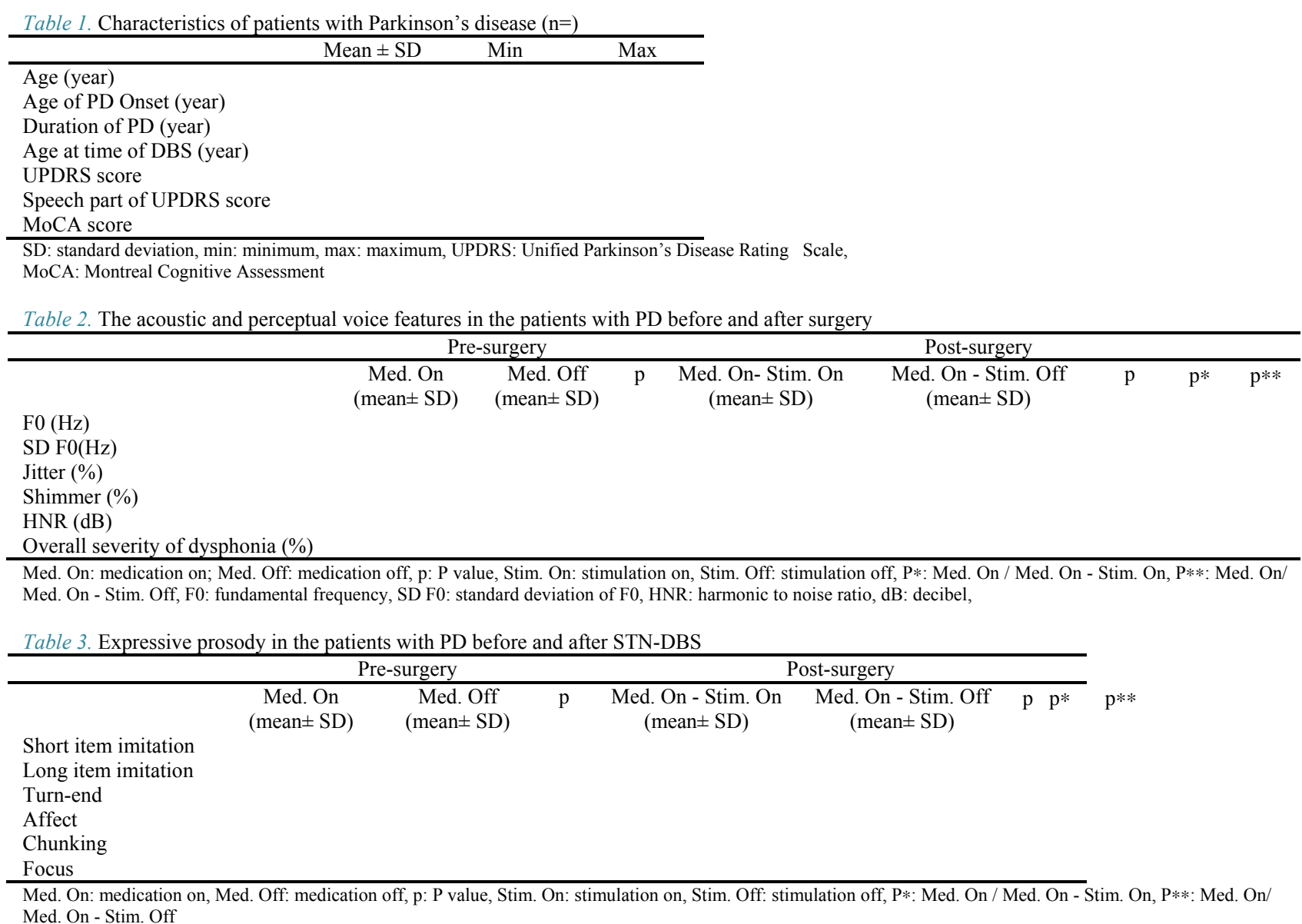

Med. On - Stim. Off

\section{Discussion}

The present study will investigate the effect of bilateral STN-DBS and levodopa therapy on the acoustic and perceptual voice features, expression of prosody and voice related quality of life in patients with PD. This is the first research to evaluate two main speech subsystems including voice and prosody disrupted by PD simultaneously. Furthermore, the efficacy of STN-DBS in Farsi speaking patients with advanced PD will be studied for the first time.

The most common treatment approaches in PD comprise pharmaceutical therapies and surgical techniques (3). Hypo dopaminergic state results in high activity of STN, basal ganglia motor circuit (BGMC) dysfunction, and reduced connectivity of the striato-thalamo-cortical motor pathways $(41,42)$. Both levodopa therapy and STN-DBS surgery resulted in better BGMC performance and more connectivity of the striato-thalamo-cortical motor pathways $(43,44)$. According to our hypothesizes, we expect to find probable improvements in the acoustic and perceptual features of voice which is associated with reduced noise, reduced dysphonia, and increased F0 variability. Moreover, it seems total scores of the PPEPS-C subtests will be increased to represent improvement ability to show different communicative intentions. All mentioned changes maybe resulted in better quality of life in patients with PD. Although the levodopa therapy will ameliorate all target voice and prosodic features even before STN-DBS under Med. On condition in regards to the dopaminergic effect of levodopa, we expect the best results can be achieved following STN-DBS under Med. On/ Stim. On condition.

Increased activity of the STN not only leads inhibitory activity of the basal ganglia on the thalamic and cortical motor system, but also leads to reduce limbic and associative system activities. Therefore, all sensorimotor, associative, and limbic loops of basal ganglia influenced by STNDBS. Due to limitation of previous studies to show the possible effect of STN-DBS on the speech characteristics of patients with PD, it seems that comprehensive evaluation of acoustic, perceptual and prosodic features of voice as well as voice related quality of life is able to demonstrate these effects more apparently. As a result, the present study will help us to better understand of pathophysiological features of sub thalamic nucleus and basal ganglia circuits, and their role in speech motor control.

\section{Conclusion}

The results of our study may show the effects of dopaminergic medications and bilateral STN DBS on the voice, expressive prosodic features, and also voice related quality of life in patients with Parkinson's disease.

\section{Limitations}

Despite of our desire, the current study will be faced to some technical difficulties. The examiner will not be 
blinded to the medication and stimulation conditions of the participants. Additionally, we will probably have some problems to find the patients who have inclusive criteria. Moreover, the surgery may be postponed due to supply necessary devices by hospital. These items can prolong the process of data gathering.

\section{Acknowledgments}

This study was part of a $\mathrm{PhD}$ dissertation supported by School of Rehabilitation, Tehran University of Medical Sciences (Grant Number: 9121363008). We wish to thank Dr. Sue Peppé who gave us much valuable advice in the early stages of this work.

\section{Conflict of Interests}

The authors declare that they have no competing interests.

\section{References}

1. Skodda, S., Aspects of speech rate and regularity in Parkinson's disease. J Neurol Sci. 2011. 310(1-2): p. 231-6.

2. Spielman, J., Mahler, L., Halpern, A., Gilley, P., Klepitskaya, O., Ramig, L., Intensive voice treatment (LSVT(R)LOUD) for Parkinson's disease following deep brain stimulation of the subthalamic nucleus. J Commun Disord. 2011. 44(6): p. 688-700.

3. Xie, Y., et al., Changes in speech characters of patients with Parkinson's disease after bilateral subthalamic nucleus stimulation. J Voice. 2011. 25(6): p. 751-758.

4. Odekerken, V.J., et al., Subthalamic nucleus versus globus pallidus bilateral deep brain stimulation for advanced Parkinson's disease (NSTAPS study): a randomised controlled trial. Lancet Neurol. 2013. 12(1): p. 37-44.

5. Ashkan, K., et al., Variability of the subthalamic nucleus: the case for direct MRI guided targeting. Br J Neurosurg. 2007. 21(2): p. 197200.

6. Parvaresh-Rizi, M., Tabibkhoei, A., Shahidi, G., Vaidyanathan, J., Tabibkhoei, A., Rohani, M., Spatial distance between anatomicallyand physiologically-identified targets in subthalamic nucleus deep brain stimulation in Parkinson's disease. Iran J Neurol. 2016. 15(1): p. 34-45.

7. Floden, D., Cooper, S.E., Griffith, S.D ., Machado,A., Predicting quality of life outcomes after subthalamic nucleus deep brain stimulation. Neurology. 2014. 83(18): p. 1627-1633.

8. Martinez-Martin, P., Rodriguez-Blazquez, C., Kurtis, M., Ray Chaudhuri,K., The impact of non-motor symptoms on health-related quality of life of patients with Parkinson's disease. J Mov Disord. 2011. 26(3): p. 399-406.

9. Kim, H.J., Jeon, B.S., Paek, S.H., Nonmotor symptoms and subthalamic deep brain stimulation in Parkinson's disease. J Mov Disord. 2015. 8(2): p. 83.

10. Mutch, W.J., Strudwickm, A., Roy, S.K., Downie, A.W., Parkinson's disease: disability, review, and management. Br Med J (Clin Res Ed), 1986. 293(6548): p. 675-7.

11. Forrest, K., Weismer, G., Turner, G.S., Kinematic, acoustic, and perceptual analyses of connected speech produced by parkinsonian and normal geriatric adults. J Acoust Soc Am. 1989. 85(6): p. 260822.

12. Majdinasab, F., Karkheiran, S., Soltani, M., Moradi, N., Shahidi, G., Relationship Between Voice and Motor Disabilities of Parkinson's Disease. J Voice. 2016. 30(6): p. 768.e17-768.e22.

13. Ho, A.K., Iansek, R., Marigliani, K., Bradshaw, J.L., Gates, S., Speech impairment in a large sample of patients with Parkinson's disease. Behav Neurol. 1998. 11(3): p. 131-137.

14. Zwirner, P., Barnes, G.J.,Vocal tract steadiness: a measure of phonatory and upper airway motor control during phonation in dysarthria. J Speech Hear Res. 1992. 35(4): p. 761-8.

15. De Bodt, M.S., Hernandez-Diaz, H.M., Van De Heyning, P.H., Intelligibility as a linear combination of dimensions in dysarthric speech. J Commun Disord. 2002. 35(3): p. 283-92.
16. Hargrove, P., McGarr, N., Prosody management of communication disorders. 1st ed. Vol. 1. 1994, San Diego,California: Singular publishing group. 1-10.

17. Skodda, S., Visser, W., Schlegel, U., Gender-related patterns of dysprosody in Parkinson disease and correlation between speech variables and motor symptoms. J Voice. 2011. 25(1): p. 76-82.

18. Benabid, A.L., Chabardes, S., Mitrofanis, J., Pollak, P., Deep brain stimulation of the subthalamic nucleus for the treatment of Parkinson's disease. Lancet Neurol. 2009. 8(1): p. 67-81.

19. Rousseaux, M., Krystkowiak, P., Kozlowski, O., Özsancak, C., Blond, S., Destée, A., Effects of subthalamic nucleus stimulation on parkinsonian dysarthria and speech intelligibility. J Neurol, 2004. 251(3): p. 327-34.

20. Dromey, C., Kumar,R., Lang, A.E., Lozano, A.M., An investigation of the effects of subthalamic nucleus stimulation on acoustic measures of voice. Movement Dis. 2000. 15(6): p. 1132-1138.

21. Gentil, M., Chauvin, P., Pinto, S., Pollak, P., Benabid, A.L., Effect of bilateral stimulation of the subthalamic nucleus on parkinsonian dysarthria. Brain Lang. 2003. 85(2): p. 190-6.

22. Tripoliti, E., et al., Effects of subthalamic stimulation on speech of consecutive patients with Parkinson disease. Neurology. 2011. 76(1): p. 80-6.

23. Skodda, S., Grönheit, W., Schlegel, U., Südmeyer, M., Schnitzler, A., Wojtecki, L., Effect of subthalamic stimulation on voice and speech in Parkinson's disease: for the better or worse? Front Neurol. 2014. 4: p. 218.

24. Tsuboi, T., et al., Distinct phenotypes of speech and voice disorders in Parkinson's disease after subthalamic nucleus deep brain stimulation. J Neurol Neurosurg Psychiatry, 2015. 86(8): p. 856-64.

25. Van Lancker Sidtis, D., Rogers, T., Godier, V., Tagliati, M., Sidtis, J.J., Voice and fluency changes as a function of speech task and deep brain stimulation. J Speech Lang Hear Res, 2010. 53(5): p. 1167-77.

26. Wertheimer, J., et al., The impact of STN deep brain stimulation on speech in individuals with Parkinson's disease :the patient's perspective. Parkinsonism Relat Disord. 2014. 20(10): p. 1065-70.

27. Péron, J., et al., Recognition of emotional prosody is altered after subthalamic nucleus deep brain stimulation in Parkinson's disease. Neuropsychologia, 2010. 48(4): p. 1053-1062

28. Bruck, C., Wildgruber, D., Kreifelts, B., Krüger, R., Wächter,T, Effects of subthalamic nucleus stimulation on emotional prosody comprehension in Parkinson's disease. PLoS One. 2011. 6(4): p. e19140.

29. Peppé, S.J., Why is prosody in speech-language pathology so difficult? Int J Speech Lang Pathol, 2009. 11(4): p. 258-271.

30. Madjdinasab, F., Moradi, N., Karkheiran, S., Kamali, M., Voice Handicap Index (VHI) in Persian speaking Parkinson's disease patients. Iranian J Age. 2014. 9(1): p. 40-45.

31. Fahn, S., Jankovic, J., Hallett, M., Principles and practice of movement disorders, ed. 2nd. 2011: Elsevier Health Sciences.

32. Athilingam, P., et al., Montreal Cognitive Assessment and MiniMental Status Examination compared as cognitive screening tools in heart failure. Heart \& Lung. J Acut Critic Care. 2011. 40(6): p. 521529.

33. Salary Majd, N., Khoddami, S.M., Drinnan, M., Kamali, M., AmiriShavaki, Y., Fallahian, N., Validity and rater reliability of Persian version of the Consensus Auditory Perceptual Evaluation of Voice. Audiol. 2014. 23(3): p. 65-74

34. Kempster, G.B., Kempster, G.B., Gerratt, B.R., Verdolini Abbott, K., Barkmeier-Kraemer, J., Hillman, R.E., Consensus auditoryperceptual evaluation of voice: development of a standardized clinical protocol. Am J Speech Lang Pathol, 2009. 18(2): p. 124-132.

35. Peppé, S., McCann, J., Gibbon, F., O'Hare, A., Rutherford, M., Assessing prosodic and pragmatic ability in children with highfunctioning autism. J Pragmat. 2006. 38(10): p. 1776-1791.

36. Moradi, N., Pourshahbaz, A., Soltani, M., Javadipour, S., Hashemi, H., Soltaninejad, N., Cross-cultural equivalence and evaluation of psychometric properties of voice handicap index into Persian. J Voice. 2013. 27(2): p .258 .e15-258. e22.

37. Jacobson, B.H., et al., The voice handicap index (VHI) development and validation. Am J Speech-Lang Path. 1997. 6(3): p. 66-70.

38. Starr, P.A., Placement of deep brain stimulators into the subthalamic nucleus or Globus pallidus internus: technical approach. Stereotact Funct Neurosurg, 2002. 79(3-4): p. 118-45.

39. Skodda, S., Rinsche, H., Schlegel, U., Progression of dysprosody in Parkinson's disease over time-a longitudinal study. Mov Disord. 
2009. 24:(5) p. 716-22.

40. Martel Sauvageau, V., et al., Articulatory Changes in Vowel Production following STN DBS and Levodopa Intake in Parkinson's Disease. Parkinsons Dis. 2015. 2015: p. 382320.

41. Higgins, E., George, M., Brain stimulation therapies for clinicians. 1391, Tehran: Arjmand. 149-150.

42. Gao, L.L., Zhang,J.R., Chan, P., Wu, T., Levodopa Effect on Basal Ganglia Motor Circuit in Parkinson's Disease. CNS Neurosci Ther. 2017. 23(1): p. 76-86.

43. Trost, M., et al., Network modulation by the subthalamic nucleus in the treatment of Parkinson's disease. Neuroimage. 2006. 31(1): p. 301-7.

44. Herz, D.M., Eickhoff, S.B., Lokkegaard, A., Siebner, H.R Functional neuroimaging of motor control in Parkinson's disease: a meta-analysis. Hum Brain Mapp. 2014. 35(7): p. 3227-37. 